\title{
Editorial
}

\section{Telemonitoring for chronic heart failure: not ready for prime time}

Juan-Pablo Casas, Joey Kwong, Shah Ebrahim

09 August 2010

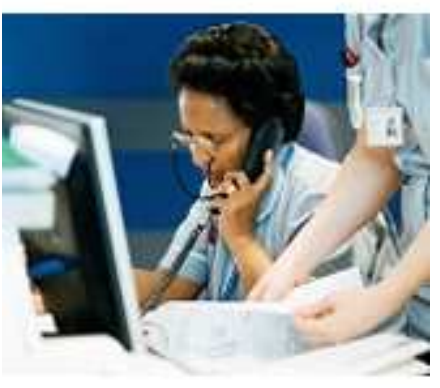

Chronic heart failure (CHF) is a life-threatening condition that is becoming more common in most countries due to improved treatments for acute coronary syndromes[1]. In addition, with ageing of the population, the number of people at risk of developing CHF has also increased. Improving the management of $\mathrm{CHF}$ is a high and growing priority for cardiovascular health services.

Multi-disciplinary team management of chronic diseases has been widely advocated, initially by geriatricians for patients with multiple pathologies. This approach has now been taken up by other specialties, although evaluations of effectiveness have been mixed [2]. Unpicking the effective components of complex interventions is challenging, and although attempts have been made to do this [3], specific trials of each component are needed to draw clear inferences. Nonetheless, finding the most 'active ingredients' of team care is an attractive proposition in low-resource settings where the full package of care may be prohibitively expensive.

The Cochrane Review by Inglis and colleagues, published in Issue 8 of the Cochrane Database of Systematic Reviews, evaluates two specific components of the multi-disciplinary approach [4]: (1) structured telephone support, which is using simple telephone technology to deliver monitoring and/or self-care management; and (2) telemonitoring, which is the electronic transmission of physiological data to a healthcare team. The review found that telemonitoring had a remarkable effect on all-cause mortality, the primary outcome: a 34\% reduction in the risk of death (95\% confidence intervals (Cl) ranging from a $19 \%$ to a $46 \%$ reduction). The effect of telephone support is less dramatic: a $12 \%$ reduction in the risk of death (which could reflect a reduction as big as $24 \%$ or an increase of $1 \%$ in mortality, $\mathrm{P}=0.08$ ). Both interventions reduced $\mathrm{CHF}$-related hospitalizations by about $20 \%$, suggesting that the effects on mortality were not achieved by increasing the use of hospital resources. Beneficial effects were also found in those trials reporting quality of life outcomes, costs of care, and acceptability to patients. Taken at face value these results make a strong case for embedding telephone support and telemonitoring into routine patient care, the conclusion drawn by the authors.

Poor reporting of duration of follow-up was a problem, making it hard to calculate numbers needed to treat to benefit (NNTB). NNTBs are helpful in decision-making around healthcare delivery where quantifying the resources required to achieve health gain is a major consideration. The authors state 
that the annual mortality from CHF ranges from 3\% to 30\%; for the NNTB, this means that between 14 and 140 patients would need to use telemonitoring to avoid one death in a year. It would be important to get a clearer idea as to which of these estimates is closest to the truth before considering widespread implementation of telemonitoring.

The authors conducted several pertinent analyses to assess the robustness of their findings. Firstly, they produced funnel plots that demonstrated some evidence of 'small study bias', with more positive results in the smaller trials. Secondly, they assessed the impact of including data from trials that had not been published as full papers on their results as a post-protocol change. When they included these trials, they found that the effect of telephone support reduced markedly (risk ratio $0.96,95 \% \mathrm{Cl} 0.84$ to 1.09). When they applied a similar approach to the analysis of telemonitoring studies, there was no change in the results.

So, how might the findings of these inconvenient analyses be handled? When funnel plots show evidence of small study bias, the search strategy should be examined for completeness, and a wider search made for unpublished findings. It is also important to consider other causes of small study bias, for example, that the smaller trials contained sicker patients that would be more likely to benefit from intervention. A standard approach for exploring causes of small study bias and dealing with them has been reported by Egger and colleagues [5], which would be valuable to follow in updates of this review.

The authors limited the inclusion of data in the meta-analyses to studies for which a full peer-reviewed publication was available as a post-protocol change. Cautious interpretation is essential when evidence has been excluded that was intended to be included at protocol stage. Further information on the outcomes of unpublished trials should be sought from abstract authors. It is not unknown for authors to attempt to 'bury' inconvenient findings in difficult-to-find journals or to fail to publish them at all. For example, one of the largest trials of the effects of cardiac rehabilitation, which found no beneficial effect, is yet to be published in a peer-reviewed journal over a decade after its completion [6].

The authors carried out a risk of bias assessment of the primary trials, but they did not use this in a sensitivity analysis comparing the higher-quality trials with the other trials. Making decisions requires explicit judgments to be made about the quality of evidence in terms of whether further research would or would not change confidence in the estimate of intervention effect [7].

Few of the trials measured quality of life outcomes and even fewer reported on costs. Telemonitoring may give false reassurance that may in turn result in adverse events, which were not considered here. There is no qualitative evidence concerning the process of care - something that should be an integral part of modern complex interventions [8]. Despite the huge success of large, simple drug trials in cardiology, expertise in designing high-quality trials of complex interventions lags behind.

The potential for such innovations to unintentionally increase health inequalities among poorer populations must be considered. The largest single trial included in this review was conducted in Argentina, indicating both the relevance and the capacity to implement these interventions in middleincome countries. India and China both expect to find technological solutions to healthcare delivery problems, and telemonitoring is an attractive option. The principles of remote management are common to a wide range of chronic diseases (e.g. hypertension, angina, diabetes), and increasing the target patient population would widen its appeal to policy-makers and patients, and probably improve cost-effectiveness. 
The authors comment on the difficulties of evaluating innovative technology in the UK National Health Service (NHS). However, unlike most health systems, the UK NHS does have the National Institute for Health and Clinical Excellence (NICE), which is the mechanism for appraisal of new technologies for use in the NHS. Telemonitoring for CHF looks ready for a NICE appraisal. On the current evidence it is likely that the verdict will be "more trials needed"

\section{Author information}

Juan-Pablo Casas $^{1}$, Joey Kwong ${ }^{2}$, and Shah Ebrahim ${ }^{3}$

${ }^{1}$ Dr Juan-Pablo Casas (juan.pablo-casas@lshtm.ac.uk), Deputy Co-ordinating Editor; ${ }^{2}$ Dr Joey Kwong (joey.kwong@lshtm.ac.uk), Managing Editor; ${ }^{3}$ Prof Shah Ebrahim (shah.ebrahim@lshtm.ac.uk), Professor of Public Health and Co-ordinating Editor; Cochrane Heart Group, Department of Epidemiology \& Population Health, London School of Hygiene \& Tropical Medicine, Keppel Street, London WC1E 7HT, UK

\section{How to cite}

Juan-Pablo Casas, Joey Kwong, Shah Ebrahim. Telemonitoring for chronic heart failure: not ready for prime time[editorial]. Cochrane Database of Systematic Reviews 2010;(8):

\subsection{2/14651858.ED000008}

\section{References}

1. Kushner FG, Smith Jr, FC, Anderson JL, Bailey SR, Blankenship JC, Green LA, et al. 2009 Focused Updates: ACC/AHA Guidelines for the Management of Patients With ST-Elevation Myocardial Infarction (Updating the 2004 Guideline and 2007 Focused Update) and ACC/AHA/SCAI Guidelines on Percutaneous Coronary Intervention (Updating the 2005 Guideline and 2007 Focused Update). Circulation 2009;120:2271-2306.

2. Smith SM, Allwright S, O'Dowd T. Effectiveness of shared care across the interface between primary and specialty care in chronic disease management. Cochrane Database of Systematic Reviews 2007, Issue 3. Art. No.: CD004910. DOI: 10.1002/14651858.CD004910.pub2.

3. Beswick AD, Rees K, Dieppe P, Ayis S, Gooberman-Hill R, Horwood J, et al. Complex interventions to improve physical function and maintain independent living in elderly people: a systematic review and meta-analysis. Lancet 2008;371(9614):725-35.

\section{Inglis SC, Clark RA, Cleland JGF, McAlister F, Stewart S. Structured telephone support or} telemonitoring programs for patients with chronic heart failure. Cochrane Database of Systematic Reviews 2010, Issue 8. Art. No.: CD007228. DOI: 10.1002/14651858.CD007228 .pub3.

5. Egger M, Sterne J, Davey Smith G. Investigating and dealing with publication and other biases in metaanalysis. BMJ 2001;323(7304):101-5.

6. West RR, Beswick AD. Mortality, morbidity and quality of life following cardiac rehabilitation: results of multicentre randomized controlled trial. Journal of the American College of Cardiology 2002;39(Suppl B):453B.

7. Guyatt GH, Oxman AD, Vist GE, Kunz R, Falck-Ytter Y, Alonso-Coello P, et al for the GRADE Working Group. GRADE: an emerging consensus on rating quality of evidence and strength of recommendations. 
BMJ 2008;336:924-6.

8. Craig P, Dieppe P, Macintyre S, Michie S, Nazareth I, Petticrew M. Developing and evaluating complex interventions: the new Medical Research Council guidance. BMJ 2008;337:a1655.

\section{Declarations of interest}

The authors have completed the Unified Competing Interest form at www.icmje.org/coi_disclosure.pdf (available upon request) and declare (1) no receipt of payment or support in kind for any aspect of the article; (2) that two authors (JP and JK) are paid by a UK National Institute for Health Research (NIHR) grant to the Cochrane Heart Group; (3) that the authors/spouse /partner/children have no financial relationships with entities that have an interest in the content of the article; and (4) that there are no other relationships or activities that could be perceived as having influenced, or that giving the appearance of potentially influencing, what was written in the submitted work.

\section{Image credit}

Mark Thomas/Science Photo Library

\section{Comments on this editorial}

\section{Evidence-informed policy decisions on remote monitoring for chronic heart failure. Is it enough?}

Francisca Garcia Lizana

10 February 2015

I strongly agree with the comments in this editorial, and would like to make further comment on the Cochrane Review by Inglis and colleagues. [1]

A problem with the evidence included in the Cochrane Review is the variability among the interventions. With regard to the structural telephone support group, the type of professional providing the support varied, as did the number of telephone contacts. For instance, one study included a telephone call each month,[2] while one study included calls on days 3, 7, 14, 21, 28 and 56.[3] The contact schedule has a direct impact on economic analysis and resource allocation, and it is currently not clear which schedule is best. The comparator groups also varied widely. Some trials used an education programme, others used different follow-up to the intervention group, some used different kinds of professionals to the intervention group, while in other studies the description of the comparator group was too brief or too vague to allow replication or comparison with the other trials. Additionally, the inclusion and exclusion criteria also varied across the trials, for example by New York Heart Association (NYHA) class, or by type of setting (post-hospital discharge or community settings). Owing to these uncertainties, and those outlined in the editorial above, are the included studies similar enough for meta-analysis? And if so, to which patients are the findings applicable? And if telemonitoring and structured telephone support are beneficial, what level of technology is appropriate? Are sophisticated and expensive home technologies appropriate in a time of crisis? And should governments invest in these technologies? The data in the current Cochrane Review are not sufficient to answer these questions. 
Since the last search date for the Cochrane Review in 2008, 20 randomized controlled trials have been published about chronic hearth failure and telemonitoring. Ten of them could possibly meet the inclusion criteria for this Cochrane Review at its next update. Only considering the largest of these 10 new relevant randomized controlled trials,[4] the result of the current Cochrane Review metanalysis may change. The large study, which included 1653 patients, found that telemonitoring did not improve the primary outcomes (readmission for any reason or death from any cause within 180 days after enrolment) or secondary outcomes (hospitalization for heart failure, number of days in the hospital, and number of hospitalizations), compared with usual care. In this trial the goal of improving the continuity of care and supporting self control was not achieved, which could be attributable to patients not using the technology sufficiently.

Policy makers live in a world with unlimited needs but limited resources, and therefore rationing of interventions may be necessary. An evidence-based approach should be taken to any rationing process, so that this sophisticated and expensive technology is given to patients who really benefit. The individual risk of every patient should be considered in a rationalized decision-making processes. More large-scale trials are required to identify six key elements relating to telephone support and telemonitoring: the best healthcare practices including the optimal frequency of follow up; the patients who benefit; the levels of staffing required; patients' views on the user friendliness of technologies; evidence on efficiency; and organization models.[5] In addition, a meta-analysis on structured telephone support versus telemonitoring should be performed.

The European Commission is promoting research via The Seven Framework Programme, which is funding innovation and development of new and sophisticated technologies. In addition, it is funding different initiatives to improve knowledge and obtain evidence for supporting a successful deployment of telemedicine services. Renewing Health is funded by the European Commission's Competitiveness and Innovation Framework Programme, and its specific aims are to analyze effectiveness through standardized outcomes; to gain knowledge on patient and user satisfaction; to provide a rigorous economic analysis; and to describe and study the organizational impact of new technologies. The European Innovation Partnership will bridge research and innovation with large-scale deployments for European Union citizens. These initiatives aim to provide evidence on a new health care system which is safe, efficient, dynamic, accessible and sustainable through information and communication technologies.

\section{References:}

1. Inglis SC, Clark RA, McAlister FA, Ball J, Lewinter C, Cullington D et al. Structured telephone support or telemonitoring programmes for patients with chronic heart failure. Cochrane Database of Systematic Reviews 2010, Issue 8. Art. No.: CD007228. DOI: 10.1002/14651858.CD007228.pub2.

2. Cleland JG, Louis AA, Rigby AS, Janssens U, Balk AH; TEN-HMS Investigators. Noninvasive home telemonitoring for patients with heart failure at high risk of recurrent admission and death: the TransEuropean Network-Home-Care Management System (TEN-HMS) study. J Am Coll Cardiol 2005;45(10):1654-64.

3. DeWalt DA, Malone RM, Bryant ME, Kosnar MC, Corr KE, Rothman RL et al. A heart failure self-management program for patients of all literacy levels: a randomized, controlled trial [ISRCTN11535170]. BMC Health Serv Res 2006;6:30.

4. Chaudhry SI, Mattera JA, Curtis JP, Spertus JA, Herrin J, Lin Z et al. Telemonitoring in patients with heart failure. N Engl J Med 2010;363(24):2301-9.

5. Garcia Lizana F, Yanez Lopez V. The information technology and communication programs management of chronic heart failure. Organizational challenges and state of the evidence. Madrid: AETS 
- Instituto de Salud Carlos III; 2009 Jan. Report No.: IPE 56/09

\section{Author information}

Seconded National Expert, Policy officer. ICT for Health Unit Directorate - General Information Society and Media. European Commission

\section{Declarations of interest}

Legal advertisement: The opinions presented are those of the author and do not necessarily represent the official view of the European Commission on the subject.

\section{Keywords}

Heart \& circulation

\section{Feedback}

To comment on this editorial or to propose ideas for future editorials please contact the Cochrane Editorial Unit (ceu@cochrane.org).

\section{About Cochrane}

\section{Publications}

\section{Community}

\section{Contact Us}

\section{WILEY}

Help \& Support

About Us

Cookies \& Privacy

Wiley Job Network

Terms \& Conditions

Advertisers \& Agents 
Powered by Wiley Online Library Copyright (C) 1999 - 2016 John Wiley \& Sons, Inc. All Rights Reserved 